\title{
Direct symbolic transformation from 3D cartesian into hyperboloidal coordinates
}

\author{
Gema María Díaz-Toca * Ioana Necula ${ }^{\dagger}$
}

November 18, 2013

\begin{abstract}
A direct transformation from cartesian coordinates into hyperboloidal coordinates (considered for biaxial hyperboloids) is presented in this paper. The transformation problem is reduced to the problem of finding the smallest positive root of a fourth degree polynomial. The analysis of the polynomial's roots is performed by an algebraically complete stratification, based on symbolic techniques (mainly Sturm-Habicht sequences and its properties related to real root counting), of a planar region situated in the positive quadrant. Two approaches for computing the polynomial's roots are presented, one based on the Merriman method and the other one obtained using the Computer Algebra System Maple. Our approach improves the solution presented in [2], being reduced to a few evaluations of symbolic expressions.
\end{abstract}

\section{Introduction}

Hyperboloidal coordinates $(\lambda, \phi, h)$ for both biaxial and triaxial hyperboloids of one sheet were first introduced in the literature in [2], together with two iteration processes for the transformation of the $3 \mathrm{D}$ cartesian coordinates $(X, Y, Z)$ of a point located, by means of the hyperboloidal height $h$, out from the hyperboloidal surface, i.e. verifying the condition

$$
\frac{X^{2}}{a^{2}}+\frac{Y^{2}}{b^{2}}-\frac{Z^{2}}{c^{2}} \geq 1
$$

into hyperboloidal coordinates. Their applications range over the Geodesy field, being of interest in hyperboloidal building and cooling tower construction.

In our paper, the hyperboloidal coordinates are considered for biaxial hyperboloids (one sheet hyperboloids of revolution around the $Z$-axis). The 3D cartesian coordinates $(X, Y, Z)$ of a point located out from a hyperboloidal surface can be expressed in terms of a latitude $\phi$, a longitude $\lambda$, and a height $h$ measured along the hyperboloidal normal (see Figure 2) as follows:

$$
\begin{aligned}
& X=(\nu+h) \cos \phi \cos \lambda, \\
& Y=(\nu+h) \cos \phi \sin \lambda, \\
& Z=\left(\nu\left(e^{2}-1\right)-h\right) \sin \phi,
\end{aligned}
$$

where (see Figures 1 and 2):

*Departamento de Matemática Aplicada, Universidad de Murcia, Spain. gemadiaz@um.es

†Departamento de Matemática Aplicada I, Universidad de Sevilla, Spain. inecula@us .es 
$-\nu$ is the prime normal section curvature:

$$
\nu=\frac{a}{\sqrt{1-e^{2} \sin ^{2} \phi}}
$$

- $e$ is the eccentricity of the hyperboloid defined in the $X-Y$ plane:

$$
e=\sqrt{1+\frac{b^{2}}{a^{2}}}
$$

- $a$ is the semi-axis of the hyperboloid defined in the $X-Y$ plane.

They are analogously defined to the geodetic coordinates by replacing the reference ellipsoid with a reference biaxial hyperboloid of one sheet. Observe that for $h=0$, the hyperboloidal coordinates satisfy the equation of the biaxial hyperboloid of one sheet

$$
\frac{X^{2}}{a^{2}}+\frac{Y^{2}}{a^{2}}-\frac{Z^{2}}{b^{2}}=1
$$

A direct transformation from cartesian coordinates into hyperboloidal coordinates is presented in our paper, by relying on the direct transformation from cartesian coordinates into geodetic coordinates presented in [6] and on the analogy between the ellipsoidal and the hyperboloidal coordinates. In addition, in the same way as in [4], symbolic techniques are used in order to analyze such transformation, thereby providing additional insights such as the complete characterization of the range of applicability.

It is important to mention that, from the user's point of view, our approach reduces the process of transformation from 3D cartesian into hyperboloidal coordinates to a few mere evaluations of symbolic expressions, and is also highly presumable to be less time consuming and more accurate than the approach presented in [2]. The arguments supporting this afirmation will be given in Section 7 .

The paper is organized as follows: the Section 2 presents Sturm-Habicht sequences and their main properties, which will be used to completely characterize the sign behaviour of the real roots of a fourth degree polynomial $P$; in the Section 3 , the polynomial $P$ is generated and an algebraic method that allows the direct symbolic transformation from $3 \mathrm{D}$ coordinates into hyperboloidal coordinates is provided; the Section 4 presents two approaches for computing the roots of the polynomial $P$, the first one based on the Merriman approach and the second one obtained using the Computer Algebra System Maple, and conjectures the algebraical equalness of the roots obtained by these approaches; the Section 5 is devoted to presenting an algebraically complete classification of a planar region situated in the positive quadrant, in terms of numbers of real roots of the polynomial $P$ which fulfill certain conditions; in the Section 6 , three examples are presented, corresponding to the three cases considered in the Section 4.1; finally, the Section 7 presents several conclusions and a further research direction.

\section{Preliminaries: The Sturm-Habicht coefficients}

In this section, the definition of the Sturm-Habicht sequence and its main properties related to the real root counting problem are introduced (for more details, see [3]).

Definition 1. Let $P$ and $Q$ be polynomials in $\mathbb{R}[x]$ and $p, q \in \mathbb{N}$, such that the polynomials' degrees are smaller than $p$ and respectively $q(\operatorname{deg}(P) \leq p$ and $\operatorname{deg}(Q) \leq q)$ :

$$
P=\sum_{k=0}^{p} a_{k} x^{k}, \quad Q=\sum_{k=0}^{q} b_{k} x^{k} .
$$




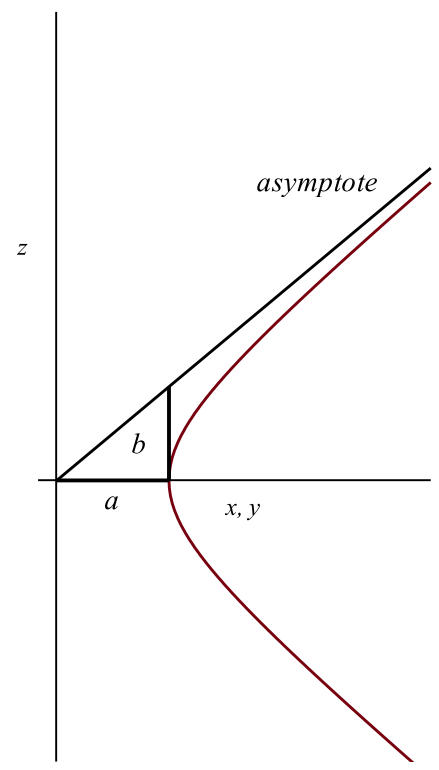

Figure 1: Fundamental geometry on the hyperbola.

If $i \in\{0, \ldots, \inf (p, q)-1\}$, then the polynomial subresultant associated to $P$ and $Q$ of index $i$ is defined as follows: $\operatorname{Sres}_{i}(P, p, Q, q)=\sum_{j=0}^{i} M_{j}^{i}(P, Q) x^{j}$, where every $M_{j}^{i}(P, Q)$ is the determinant of the matrix built with the columns $1,2, \ldots, p+q-2 i-1$ and $p+q-i-j$ in the matrix:

$$
m_{i}(P, p, Q, q)=\overbrace{\left(\begin{array}{ccccc}
a_{p} & \ldots & a_{0} & & \\
& \ddots & & \ddots & \\
& & a_{p} & \ldots & a_{0} \\
b_{q} & \ldots & b_{0} & & \\
& \ddots & & \ddots & \\
& & b_{q} & \ldots & b_{0}
\end{array}\right)}^{p+q-i}\} q-i
$$

The determinant $M_{i}^{i}(P, Q)$ will be called the $i^{\text {th }}$ principal subresultant coefficient and denoted by $\operatorname{sres}_{i}(P, p, Q, q)$.

The following definition introduces the Sturm-Habicht sequence associated to $P$ and $Q$ as the sequence of the subresultants associated to $P$ and $P^{\prime} Q$ modulo certain sign changes.

Definition 2. Let $P$ and $Q$ be polynomials in $\mathbb{R}[x]$ such that $p=\operatorname{deg}(P)$ and $q=$ $\operatorname{deg}(Q)$. Let $v=p+q-1$ and $\delta_{k}=(-1)^{\frac{k(k+1)}{2}}$ for $k \in \mathbb{N}$. The Sturm-Habicht sequence associated to $P$ and $Q$ is defined as the polynomial list $\left\{\mathbf{S t H a}_{j}(P, Q)\right\}_{j=0, \ldots, v+1}$ where $\mathbf{S t H a}_{v+1}(P, Q)=P, \mathbf{S t H a}_{v}(P, Q)=P^{\prime} Q$, and for every $j \in\{0, \ldots, v-1\}$ :

$$
\operatorname{StHa}_{j}(P, Q)=\delta_{v-j} \operatorname{Sres}_{j}\left(P, v+1, P^{\prime} Q, v\right) .
$$

For every $j \in\{0, \ldots, v+1\}$, the $j^{\text {th }}$ Sturm-Habicht principal coefficient is defined as follows:

$$
\operatorname{stha}_{j}(P, Q)=\operatorname{coef}_{j}\left(\mathbf{S t H a}_{j}(P, Q)\right),
$$

i.e. the coefficient of $x^{j}$ in the polynomial $\mathbf{S t H a} \mathbf{a}_{j}(P, Q)$. 
Remark 1. In the particular case of $Q=1$, the Sturm-Habicht sequence associated to $P$ are denoted by $\mathbf{S t H a}_{j}(P)$, and the Sturm-Habicht principal coefficients associated to $P$ by $\operatorname{stha}_{j}(P)$.

In order to establish the relation between the real roots of a polynomial $P$ in $\mathbb{R}[x]$ and the polynomials appearing in the Sturm-Habicht sequence associated to $P$ and $Q$ (with $Q \in \mathbb{R}[x])$, the following integer numbers are introduced:

$$
c_{[\epsilon]}(P, Q)=\#\{\alpha \in \mathbb{R}: P(\alpha)=0, \operatorname{sign}(Q(\alpha))=\epsilon\}, \quad \epsilon \in\{+,-, 0\} .
$$

By studying the sign changes of Sturm-Habicht principal coefficients, highly useful information about the number of real roots of the considered polynomial can be obtained. The following definitions present the sign change functions which allow the number of real roots of a polynomial in $\mathbb{R}[x]$ to be computed.

Definition 3. Let $I=\left\{a_{0}, a_{1}, \ldots, a_{n}\right\}$ be a list of real, non-zero numbers

- $\mathbf{V}(I)$ represents the number of sign variations in the list $\left\{a_{0}, a_{1}, \ldots, a_{n}\right\}$ :

$$
\mathbf{V}(I)= \begin{cases}0 & \text { if } n=0 \\ \mathbf{V}\left(\left\{a_{0}, \ldots, a_{n-1}\right\}\right)+1 & \text { if } \operatorname{sign}\left(a_{n} a_{n-1}\right)=-1 \\ \mathbf{V}\left(\left\{a_{0}, \ldots, a_{n-1}\right\}\right) & \text { otherwise }\end{cases}
$$

- $\mathbf{P}(I)$ represents the number of sign permanences in the list $\left\{a_{0}, a_{1}, \ldots, a_{n}\right\}$ :

$$
\mathbf{P}(I)= \begin{cases}0 & \text { if } n=0 \\ \mathbf{P}\left(\left\{a_{0}, \ldots, a_{n-1}\right\}\right)+1 & \text { if } \operatorname{sign}\left(a_{n} a_{n-1}\right)=1 \\ \mathbf{P}\left(\left\{a_{0}, \ldots, a_{n-1}\right\}\right) & \text { otherwise }\end{cases}
$$

The previous definition can be extended to lists of any real numbers (not necessarily non-zero numbers). If $I_{0}$ is the list obtained by eliminating all the zeros from a list $I$, then $\mathbf{V}(I):=\mathbf{V}\left(I_{0}\right)$ and $\mathbf{P}(I):=\mathbf{P}\left(I_{0}\right)$ are defined.

Notation 1. Let $\left\{a_{0}, a_{1}, \ldots, a_{n}\right\} \subset \mathbb{R}$, with $a_{0} \neq 0$ and with the following zero distribution:

$$
\begin{gathered}
I=\left\{a_{0}, a_{1}, \ldots, a_{n}\right\}= \\
=\{a_{0}, \ldots, a_{i_{1}}, \overbrace{0, \ldots, 0}^{k_{1}}, \overbrace{a_{i_{1}+k_{1}+1}, \ldots, a_{i_{2}}, \overbrace{0, \ldots, 0}^{k_{2}}, a_{i_{2}+k_{2}+1}, \ldots, a_{i_{3}}, \ldots, \overbrace{0, \ldots, 0}^{k_{t}},}^{k_{t-1}}, \overbrace{0, \ldots, 0}^{k_{i_{t-1}+k_{t-1}+1}, \ldots, a_{i_{t}}, \ldots, \ldots}\}
\end{gathered}
$$

where all the written elements $a_{i}$ are different from 0 . Denoting $i_{0}+k_{0}+1=0$, define $\mathbf{C}(I)$ by

$$
\mathbf{C}(I)=\sum_{s=1}^{t}\left(\mathbf{P}\left(\left\{a_{i_{s-1}+k_{s-1}+1}, \ldots, a_{i_{s}}\right\}\right)-\mathbf{V}\left(\left\{a_{i_{s-1}+k_{s-1}+1}, \ldots, a_{i_{s}}\right\}\right)\right)+\sum_{s=1}^{t-1} \varepsilon_{i_{s}}
$$

where:

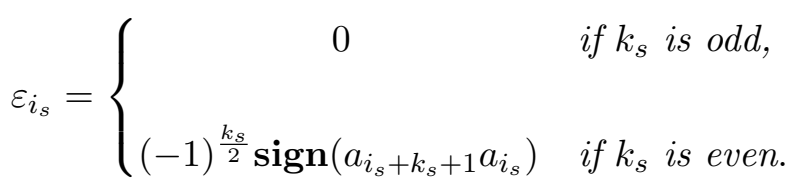

The relation between the number of real roots of a polynomial $P \in \mathbb{R}[x]$ and the polynomial appearing in the Sturm-Habicht sequence associated to $P$ is stated below. 
Theorem 1. If $P$ and $Q$ are polynomials in $\mathbb{R}[x]$ then:

$$
\Omega(P, Q):=\mathbf{C}\left(\left\{\operatorname{stha}_{p}(P, Q), \ldots, \operatorname{stha}_{0}(P, Q)\right\}\right)=c_{[+]}(P, Q)-c_{[-]}(P, Q) .
$$

If $P$ and $Q$ are polynomials in $\mathbb{R}[x]$ and have no common real root, then (see $[1]) c_{[+]}(P, Q)$ and $c_{[-]}(P, Q)$ can be easily computed in terms of $\Omega(P, Q)$ and $\Omega(P, 1)$ :

$$
\begin{aligned}
& c_{[+]}(P, Q)=\frac{\Omega(P, 1)+\Omega(P, Q)}{2} \\
& c_{[-]}(P, Q)=\frac{\Omega(P, 1)-\Omega(P, Q)}{2}
\end{aligned}
$$

\section{Transformation from cartesian into hyperboloidal coordinates}

As mentioned in the Introduction, the 3D cartesian coordinates are related to the hyperboloidal coordinates by (see Figure 2)

$$
\begin{aligned}
& X=(\nu+h) \cos \phi \cos \lambda, \\
& Y=(\nu+h) \cos \phi \sin \lambda, \\
& Z=\left(\nu\left(e^{2}-1\right)-h\right) \sin \phi,
\end{aligned}
$$

where

$$
\nu^{2}=\frac{a^{2}}{1-e^{2} \sin ^{2} \phi}
$$

In the following, an algebraic method that allows the direct symbolic transformation from $3 \mathrm{D}$ coordinates into hyperboloidal coordinates is provided. Observe that the case $Z=0$ is trivial: if $Z=0$, then $\phi=0, \nu=a$, and $h=\sqrt{X^{2}+Y^{2}}-a$. Therefore, the conditions $Z>0, \sin (\phi)>0$ and $\cos (\phi)>0$ are henceforth supposed to be verified. Obviously this implies that $\nu\left(e^{2}-1\right)-h>0$.

Let the positive coefficient $k$ be defined as follows:

$$
k:=\frac{\nu\left(e^{2}-1\right)-h}{\nu}>0,
$$

Hence,

$$
h=\nu\left(e^{2}-1\right)-k \nu=\nu\left(e^{2}-k-1\right) \geq 0,
$$

and from (3) and (7),

$$
Z=k \nu \sin \phi
$$

consequently,

$$
\nu^{2}=\frac{a^{2}}{1-e^{2} \sin ^{2} \phi}=\frac{a^{2}}{1-\frac{e^{2} Z^{2}}{k^{2} \nu^{2}}}=\frac{k^{2} \nu^{2} a^{2}}{k^{2} \nu^{2}-e^{2} Z^{2}}
$$

and therefore

$$
\nu^{2}=a^{2}+\frac{e^{2} Z^{2}}{k^{2}} \Rightarrow \nu=\sqrt{a^{2}+\frac{e^{2} Z^{2}}{k^{2}}} .
$$

Furthermore, from (1) and (2),

$$
X^{2}+Y^{2}=(\nu+h)^{2} \cos ^{2} \phi=\nu^{2}\left(e^{2}-k\right)^{2} \cos ^{2} \phi=\nu^{2}\left(e^{2}-k\right)^{2}\left(1-\frac{Z^{2}}{k^{2} \nu^{2}}\right)=
$$




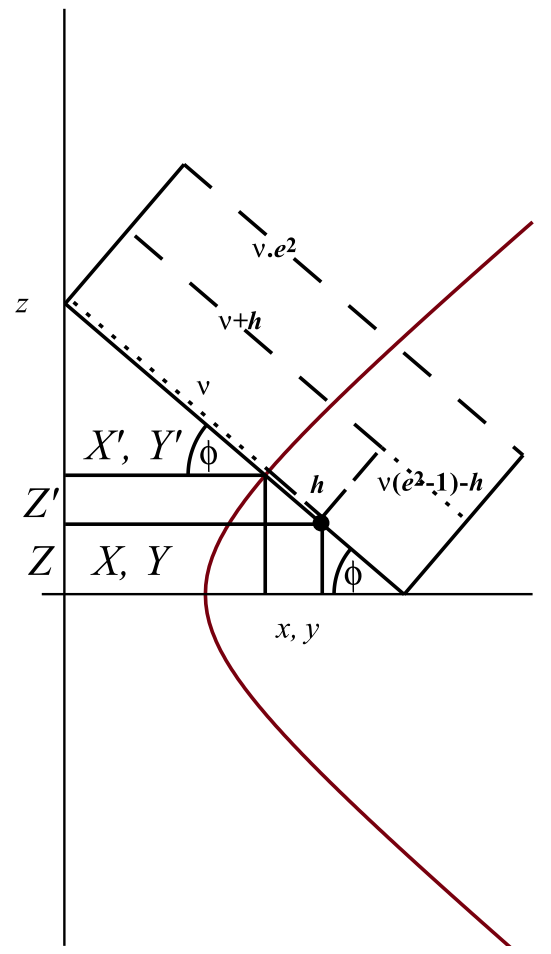

Figure 2: Distances $\nu, \nu e^{2}$ and $\nu\left(e^{2}-1\right)-h$ on the hyperbola, and the definition of the hyperboloidal height $h$.

$$
=\left(e^{2}-k\right)^{2} \frac{a^{2} k^{2}+e^{2} Z^{2}-Z^{2}}{k^{2}}
$$

and therefore

$$
\frac{X^{2}+Y^{2}}{\left(e^{2}-k\right)^{2}}-\frac{Z^{2}\left(e^{2}-1\right)}{k^{2}}=a^{2}
$$

On the other hand, by defining two new variables $p$ and $q$ as follows:

$$
p:=\frac{X^{2}+Y^{2}}{a^{2}}>0, \quad q:=\frac{e^{2}-1}{a^{2}} Z^{2}>0,
$$

the following formula can be deduced from (10) :

$$
\frac{p}{\left(e^{2}-k\right)^{2}}-\frac{q}{k^{2}}=1
$$

that is,

$$
p k^{2}-\left(e^{2}-k\right)^{2} q=k^{2}\left(e^{2}-k\right)^{2} .
$$

Through expansion, a degree 4 univariate polynomial (in $k$ ), whose coefficients depend on $e, p$ and $q$, is obtained:

$$
P(e, p, q ; k):=k^{4}-2 e^{2} k^{3}-\left(p-q-e^{4}\right) k^{2}-2 e^{2} q k+q e^{4}=0
$$

Taking into account the generating process of the polynomial $P(e, p, q ; k)$, there must be a positive real root $k$ such that, given $(X, Y, Z)$, the distance $D:=\left(\nu\left(e^{2}-1\right)-h\right) \cos \phi$ 
(see Figure 3) can be calculated by using Equation (9):

$$
D=\left(\nu\left(e^{2}-1\right)-h\right) \cos \phi=k \nu \cos \phi=\frac{k \sqrt{X^{2}+Y^{2}}}{e^{2}-k}>0 .
$$

Observe that, since $h \geq 0, k$ must verify the condition $e^{2}-k \geq 1$ (see (8)), and therefore the denominator of $D$ is positive.

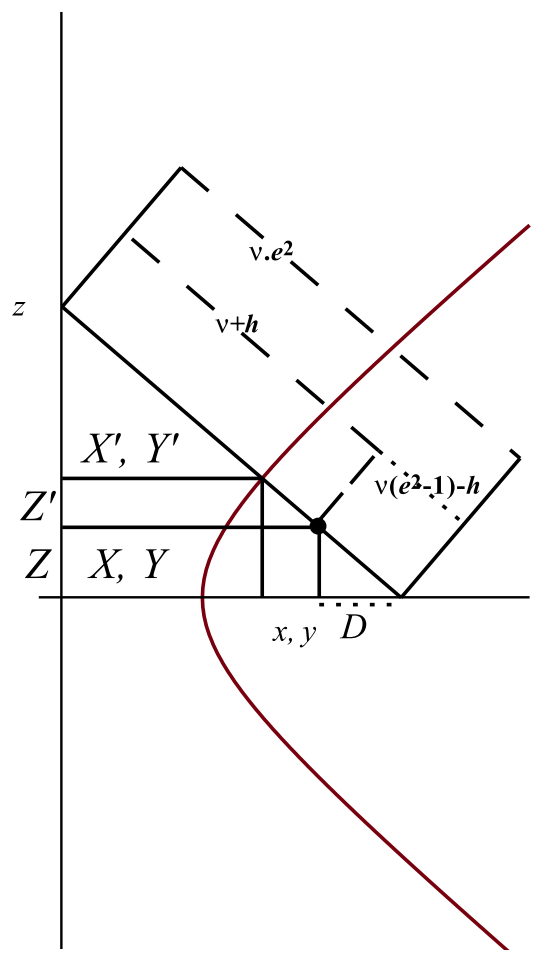

Figure 3: Distance $D$.

Once $D$ is computed, the hyperboloidal coordinates $(\lambda, \phi, h)$ can be computed from $(7)$ and (10) as follows:

$$
\begin{gathered}
\nu=\sqrt{a^{2}+\frac{e^{2} Z^{2}}{k^{2}}}=\sqrt{\frac{X^{2}+Y^{2}}{\left(e^{2}-k\right)^{2}}+\frac{Z^{2}}{k^{2}}}=\frac{\sqrt{D^{2}+Z^{2}}}{k}, \\
h=\nu\left(e^{2}-1-k\right)=\frac{\sqrt{D^{2}+Z^{2}}\left(e^{2}-1-k\right)}{k} \\
\lambda=\arctan \left(\frac{Y}{X}\right) \\
\tan \frac{\phi}{2}=\frac{\sin \phi}{1+\cos \phi}=\frac{Z}{D+\sqrt{D^{2}+Z^{2}}} .
\end{gathered}
$$

Remark 2. The conditions $p>0$ and $q>0$ are supposed to be verified. Observe that:

- $Z \neq 0$ implies $q>0$;

- $p=0$ would imply $X=Y=0$ and in this case the point $(X, Y, Z)$ would be on the Z-axis. 
Remark 3. Let $\Delta_{0}(e ; p, q)$ denote the polynomial $\left(e^{2}-1\right)^{2} p-q-\left(e^{2}-1\right)^{2}$. Due to the geometry of the problem (the point given in $3 D$ cartesian coordinates is located out from the hyperboloidal surface), the following condition holds (see (11) and (4)):

$$
\Delta_{0}(e ; p, q) \geq 0
$$

The case of a point located on the hyperboloidal surface (whose cartesian coordinates satisfy the equation (5)), or equivalently the equation $\Delta_{0}(e ; p, q)=0$, is trivial. It is very easy to prove that in this case the hyperboloidal coordinates $(\lambda, \phi, h)$ are:

$$
\begin{aligned}
h & =0 \\
\lambda & =\arctan \left(\frac{Y}{X}\right) \\
\tan \frac{\phi}{2} & =\frac{Z}{\left(e^{2}-1\right)\left(\nu+\sqrt{X^{2}+Y^{2}}\right)} .
\end{aligned}
$$

Furthermore, the condition

$$
\frac{X^{2}}{a^{2}}+\frac{Y^{2}}{a^{2}}-\frac{Z^{2}}{b^{2}}>1
$$

which is equivalent to

$$
\Delta_{0}(e ; p, q)>0
$$

is supposed to be verified.

\section{Computation of $P(e, p, q ; k)$ 's roots}

In this section, two approaches for computing the roots of the polynomial $P(e, p, q ; k)$ are presented. The first one was introduced at the ends of the $19^{\text {th }}$ century by M. Merriman, meanwhile the second one is based on using symbolic computation tools of the Computer Algebra System Maple, and followed by a post-processing step of manually performed simplification.

\subsection{Computation of $P(e, p, q ; k)$ 's roots based on Merriman's approach}

In order to compute the roots of $P(e, p, q ; k)$, by using the approach in [5] and keeping its notation, the following parameters are determined:

$$
\begin{aligned}
N & :=\frac{\left(e^{4}+q-p\right)^{6}}{6^{6}} \\
M & :=\frac{\left(e^{4}+q-p\right)^{3}+54 e^{4} p q}{6^{3}} \\
S & :=\frac{\sqrt[3]{M+\sqrt{M^{2}-N}}}{2} \\
T & :=\frac{\sqrt[3]{M-\sqrt{M^{2}-N}}}{2} \\
u & :=\frac{e^{4}+2 p-2 q}{12}+(S+T) \\
v & :=\frac{e^{4}+2 p-2 q}{6}-(S+T) \\
w & :=v^{2}+3(S-T)^{2} .
\end{aligned}
$$


By considering that $y_{1}, y_{2}$ and $y_{3}$ are the roots of the cubic

$$
y^{3}-\frac{e^{4}+2 p-2 q}{4} y^{2}+\frac{(p-q)\left(p-q+2 e^{4}\right)}{16} y-\frac{e^{4}(p+q)^{2}}{64}=0,
$$

and being $y_{1}$ a real root ( $y_{2}$ and $y_{3}$ may be real or complex conjugated roots), it follows that

$$
\begin{aligned}
y_{1} & =u \in \mathbb{R} \\
y_{2}+y_{3} & =v \in \mathbb{R} \\
4 y_{2} y_{3} & =w \in \mathbb{R}
\end{aligned}
$$

(for more details, see [5]). Observe that $(S-T)^{2}$ can be positive or negative (depending on the sign of $\left.M^{2}-N\right)$.

Remark 4. It is important to mention that if $M^{2}-N<0$, then:

- the expression $\sqrt{M^{2}-N}$ stands for the complex number $I \sqrt{N-M^{2}}$, and

- the expression $\sqrt[3]{M+\sqrt{M^{2}-N}}$ stands for the cubic complex root $\psi+I \omega$ verifying the conditions $\psi>0$ and $\omega>0$.

In the following, the existence of the aforementioned cubic complex root $\psi+I \omega$ is proved (when $\left.M^{2}-N<0\right)$. The complex number $M+\sqrt{M^{2}-N}=M+I \sqrt{N-M^{2}}$ can be written also as $\rho(\cos (\theta)+I \sin (\theta)$, with $\rho=\sqrt{N}$ and

$$
\left.\cos (\theta)=\frac{M}{\sqrt{N}}, \quad \sin (\theta)=\sqrt{\frac{N-M^{2}}{N}}>0 \text { (hence, } \theta \in(0, \pi)\right) .
$$

By applying the De Moivre theorem, the equation $z^{3}=M+I \sqrt{N-M^{2}}$ has the cubic complex roots:

$$
z_{j}=\sqrt[3]{\rho}\left[\cos \left(\frac{\theta+2 j \pi}{3}\right)+I \sin \left(\frac{\theta+2 j \pi}{3}\right)\right], \quad j=0,1,2 .
$$

The root of interest from our point of view is $z_{0}=\psi+I \omega$, as (see (23))

$$
\cos \left(\frac{\theta}{3}\right)>0, \quad \sin \left(\frac{\theta}{3}\right)>0 .
$$

The four roots of $P(e, p, q ; k)$ are thus presented as:

$$
\begin{aligned}
& \alpha_{1}:=\frac{e^{2}}{2}+\sqrt{u}+\sqrt{v+\sqrt{w}}, \\
& \alpha_{2}:=\frac{e^{2}}{2}+\sqrt{u}-\sqrt{v+\sqrt{w}}, \\
& \alpha_{3}:=\frac{e^{2}}{2}-\sqrt{u}+\sqrt{v-\sqrt{w}}, \\
& \alpha_{4}:=\frac{e^{2}}{2}-\sqrt{u}-\sqrt{v-\sqrt{w}} .
\end{aligned}
$$

In the following, the nature of these roots in the region of interest for us (that is, when $p>0, q>0$ and $\left.\Delta_{0}(e ; p, q)>0\right)$ is examined, by means of the behaviour of the resultant of $P(e, p, q ; k)$ and its derivative with respect to $k$ (ignoring constant factors), denoted by $\Delta_{3}(e ; p, q)$,

$$
\Delta_{3}(e ; p, q)=p^{3}-3 p^{2} q-3 e^{4} p^{2}+3 p q^{2}-21 e^{4} q p+3 e^{8} p-3 e^{8} q-q^{3}-3 e^{4} q^{2}-e^{12} .
$$


Observe that

$$
\begin{aligned}
\Delta_{3}(e ; p, q) & =\left(p-q-e^{4}\right)^{3}-27 e^{4} p q, \\
M & =\frac{27 e^{4} p q-\Delta_{3}(e ; p, q)}{6^{3}}
\end{aligned}
$$

and the signs of $M^{2}-N$ and $\Delta_{3}(e ; p, q)$ are opposite, as

$$
M^{2}-N=-\frac{e^{4} p q}{432} \Delta_{3}(e ; p, q) .
$$

Moreover, if $\Delta_{1}(e ; p, q)$ denotes the polynomial $2 p-2 q+e^{4}$, then

$$
\begin{aligned}
& u=\frac{\Delta_{1}(e ; p, q)}{12}+(S+T), \\
& v=\frac{\Delta_{1}(e ; p, q)}{6}-(S+T) .
\end{aligned}
$$

In the following the three cases $\Delta_{3}(e ; p, q)<0, \Delta_{3}(e ; p, q)=0$ and $\Delta_{3}(e ; p, q)>0$ are considered.

First, the case $\Delta_{3}(e ; p, q)<0$ is considered. It follows that $M^{2}-N>0, M>0$ and therefore $S$ and $T$ are both positive real numbers. Then $\sqrt{w}$ is a real number, $v+\sqrt{w}>0$ and $v-\sqrt{w}<0$. With respect to the real number $u$, it is easy to see that if $\Delta_{1}(e ; p, q) \geq 0$, then $\sqrt{u}$ is a real number. Otherwise, if $\Delta_{1}(e ; p, q)<0$, the Descartes rule applied to the cubic (19) asserts that $u$ is also positive (observe that the coefficient of $y^{2}$ in (19) is $\left.-\frac{1}{4} \Delta_{1}(e ; p, q)\right)$. In conclusion, $\alpha_{1}$ and $\alpha_{2}$ are the two positive roots and $\alpha_{3}$ and $\alpha_{4}$ are the complex conjugated roots.

Next, the case $\Delta_{3}(e ; p, q)=0$ is considered. It follows that $M^{2}-N=0, M>0$, $S=T=\frac{1}{2} \sqrt[3]{M}>0, w=v^{2}$. Moreover since $p-q-e^{4}>0, \Delta_{1}(e ; p, q)>0$ and therefore $u>0$. Under these hypotheses, $v$ must be positive. Therefore $\alpha_{1}$ and $\alpha_{2}$ are the two different positive roots and $\alpha_{3}=\alpha_{4}$ is the negative double root.

Finally, the case $\Delta_{3}(e ; p, q)>0$ is considered. On the one hand the roots $y_{2}$ and $y_{3}$ of the cubic (19) are real (see [5]) and the Descartes rule asserts that in this case, the three roots, $y_{1}=u, y_{2}$ and $y_{3}$ must be positive (observe that the coefficient of $y$ is positive), so that $u, v$ and $w$ are positive. On the other hand, it follows that $\Delta_{1}(e ; p, q)>0, M^{2}-N<0$, and $S$ and $T$ are complex conjugated numbers. In this case $(S-T)^{2}$ is negative and consequently $v \pm \sqrt{w}>0$. In the following, it will be proved that $\alpha_{2}>\alpha_{3}$. Let $a$ and $b$ be the real and respectively the imaginary part of $S$. Therefore $M+I \sqrt{N-M^{2}}=8(a+I b)^{3}$, which implies that $N-M^{2}=8 b\left(3 a^{2}-b^{2}\right)=$ $8 b(\sqrt{3} a+b)(\sqrt{3} a-b)$ and consequently (see Remark 4) $\sqrt{3} a-b$ is positive. It is very easy to prove that $\alpha_{2}>\alpha_{3}$ if and only if $2 u-v>\sqrt{v^{2}-w}$. As $2 u-v=3(S+T)=6 a$, $\sqrt{v^{2}-w}=\sqrt{-3(S-T)^{2}}=\sqrt{12 b^{2}}=2 \sqrt{3} b$ and $\sqrt{3} a>b$, the previous condition is verified. As a consequence, $\alpha_{1}$ and $\alpha_{2}$ are the two positive roots and $\alpha_{3}$ and $\alpha_{4}$ are the two negative roots.

Thus, the following conclusions can be drawn:

- The polynomial $P(e, p, q ; k)$ has two different positive real roots $\alpha_{1}$ and $\alpha_{2}\left(\alpha_{1}>\alpha_{2}\right)$.

- If $\Delta_{3}(e ; p, q)<0$, then $P(e, p, q ; k)$ has two complex roots $\alpha_{3}$ and $\alpha_{4}$.

- If $\Delta_{3}(e ; p, q)=0$, then $P(e, p, q ; k)$ has a double negative root $\alpha_{3}=\alpha_{4}$.

- If $\Delta_{3}(e ; p, q)>0$, then $P(e, p, q ; k)$ has two negative roots $\alpha_{3}$ and $\alpha_{4}\left(\alpha_{3}>\alpha_{4}\right)$. 


\subsection{Computation of $P(e, p, q ; k)$ 's roots via Maple}

The Computer Algebra System Maple generically computes (i.e. expresses in terms of $p, q$ and $e)$ the roots of the polynomial $P(p, q, e ; k)$ applying the Ferrari method. By accomplishing a post-processing step of manually performed simplification, the parameters $\alpha$ and $\beta$ are defined as follows:

$$
\begin{aligned}
\alpha & :=\sqrt[3]{\left(3 \sqrt{3 p q} e^{2}+\sqrt{-\Delta_{3}(e ; p, q)}\right)^{2}} \\
\beta & :=\frac{1}{3}\left(\Delta_{1}(e ; p, q)+\alpha+\frac{\left(p-q-e^{4}\right)^{2}}{\alpha}\right) .
\end{aligned}
$$

Remark 5. If $\Delta_{3}(e ; p, q)>0$, then the expression $\sqrt[3]{\left(3 \sqrt{3 p q} e^{2}+\sqrt{-\Delta_{3}(e ; p, q)}\right)^{2}}$ stands for that cubic complex root verifying that its real and imaginary parts are positive. The proof of the existence of this cubic root is similar to the one presented in Section 4.1.

The four roots of the polynomial $P$ are presented in the following way:

$$
\begin{aligned}
& k_{1}:=\frac{1}{2}\left(e^{2}+\sqrt{\beta}+\sqrt{\Delta_{1}(e ; p, q)-\beta+\frac{2 e^{2}(p+q)}{\sqrt{\beta}}}\right), \\
& k_{2}:=\frac{1}{2}\left(e^{2}+\sqrt{\beta}-\sqrt{\Delta_{1}(e ; p, q)-\beta+\frac{2 e^{2}(p+q)}{\sqrt{\beta}}}\right) \\
& k_{3}:=\frac{1}{2}\left(e^{2}-\sqrt{\beta}+\sqrt{\Delta_{1}(e ; p, q)-\beta-\frac{2 e^{2}(p+q)}{\sqrt{\beta}}}\right) \\
& k_{4}:=\frac{1}{2}\left(e^{2}-\sqrt{\beta}-\sqrt{\Delta_{1}(e ; p, q)-\beta-\frac{2 e^{2}(p+q)}{\sqrt{\beta}}}\right) .
\end{aligned}
$$

Thus, the following result is conjectured: $k_{i}=\alpha_{i}$ for every $i \in\{1,2,3,4\}$, and the challenge to prove it is left for the readers.

\section{Symbolic analysis of the positive roots of $P$}

The previous section asserts that the polynomial $P$ has two different positive roots. In this section, the Sturm-Habicht coefficients and their properties are used to certify that there is only one positive root of $P$ which makes $h=\nu\left(e^{2}-1-k\right)$ positive. Thus, the greatest positive root $\alpha_{1}$ must be discarded, being of interest from the geometrical point of view only the smallest positive root $\alpha_{2}$.

As proven in Section 4.1 (see (25)), $\alpha_{1}+\alpha_{2}=e^{2}+2 \sqrt{u}$, where $u$ is a positive real number. The generating process of the polynomial $P(e, p, q ; k)$ assures the existence of (at least) one positive real root $k$ which makes $h$ positive. If both $\alpha_{1}$ and $\alpha_{2}$ made $h$ positive, then $e^{2}+2 \sqrt{u}=\alpha_{1}+\alpha_{2} \leq 2 e^{2}-2$ or equivalently $2 \sqrt{u} \leq e^{2}-2$, which is impossible if $e<\sqrt{2}$. Thus, if $e<\sqrt{2}$ the existence of a unique positive real root of $P$ which makes $h$ positive is certified. In the following, the case $e \geq \sqrt{2}$ is tackled.

The Sturm-Habicht coefficients and their properties are used in order to stratify the space of the parameters $p>0, q>0$, verifying also the condition (18) (by considering that the eccentricity of the hyperboloid defined in the $X-Y$ plane is an arbitrary given value $e \geq \sqrt{2}$ ), in sets (regions, curves or points) where the polynomial $P$ (as a polynomial in $k$ ) has a constant number of real roots (counted without multiplicities) making $h$ positive. 
For this purpose, the polynomial $Q(e ; k):=-k+e^{2}-1$ is considered. The stratification is performed by applying formulae (6) for polynomials $P$ and $Q$ (as polynomials in $k$ ). For a formal exposition of the stratification (and generally of decompositions of sets defined by polynomial inequalities), see [1].

The Sturm-Habicht coefficients associated to $P$ are:

$$
\begin{aligned}
& \operatorname{stha}_{4}(P)=1 \\
& \operatorname{stha}_{3}(P)=4 \\
& \operatorname{stha}_{2}(P)=4\left(2 p-2 q+e^{4}\right) \\
& \operatorname{stha}_{1}(P)=8\left(p^{3}-3 p^{2} q-e^{8} q+3 p q^{2}+e^{8} p-2 e^{4} q^{2}-14 e^{4} q p-q^{3}-2 e^{4} p^{2}\right) \\
& \operatorname{stha}_{0}(P)=16 e^{4} p\left(p^{3}-3 p^{2} q-3 e^{4} p^{2}+3 p q^{2}-21 e^{4} q p+3 e^{8} p-3 e^{8} q-q^{3}-3 e^{4} q^{2}-e^{12}\right) .
\end{aligned}
$$

The Sturm-Habicht coefficients associated to $P$ and $Q$ are:

$$
\begin{aligned}
\operatorname{stha}_{5}(P, Q)= & 0 \\
\operatorname{stha}_{4}(P, Q)= & -4 \\
\operatorname{stha}_{3}(P, Q)= & 8\left(2-e^{2}\right) \\
\operatorname{stha}_{2}(P, Q)= & 16\left(p^{2}-2 p q+q^{2}-2 p-e^{2} p+2 e^{4} p+2 q-5 e^{2} q+e^{4} q+e^{6}-e^{4}\right) \\
\operatorname{stha}_{1}(P, Q)= & 32\left(e^{2} p^{4}-p^{4}+4 p^{3} q-3 e^{2} p^{3} q+3 e^{2} p^{2} q^{2}-6 p^{2} q^{2}+4 p q^{3}-e^{2} p q^{3}-q^{4}\right. \\
& +2 e^{4} p^{3}+p^{3}-e^{2} p^{3}-2 e^{6} p^{3}+4 e^{2} p^{2} q+14 e^{4} p^{2} q-3 p^{2} q-20 e^{6} p^{2} q \\
& -13 e^{4} p q^{2}-5 e^{2} p q^{2}-5 e^{6} p q^{2}+3 p q^{2}-q^{3}-3 e^{4} q^{3}+2 e^{2} q^{3}+e^{10} p^{2} \\
& -2 e^{4} p^{2}+2 e^{6} p^{2}-e^{8} p^{2}+e^{8} p q-14 e^{4} p q-4 e^{10} p q+21 e^{6} p q+4 e^{6} q^{2} \\
& \left.-3 e^{8} q^{2}-2 e^{4} q^{2}+e^{8} p-e^{10} p-e^{12} q+2 e^{10} q-e^{8} q\right) \\
\operatorname{stha}_{0}(P, Q)= & 64 p q e^{4}\left(\left(e^{2}-1\right)^{2} p-q-\left(e^{2}-1\right)^{2}\right)\left(p^{3}-3 p^{2} q-3 e^{4} p^{2}+3 p q^{2}\right. \\
& \left.-21 e^{4} q p+3 e^{8} p-3 e^{8} q-q^{3}-3 e^{4} q^{2}-e^{12}\right) .
\end{aligned}
$$

It is easy to prove, by algebraic manipulation of its coefficients, that $\operatorname{stha}_{2}(P, Q)>0$ when the conditions $p>0, q>0$ and $\Delta_{0}(e ; p, q)>0$ hold. After simplifying the aforementioned Sturm-Habicht coefficients, the conclusion that $\Omega(P, 1)$ and $\Omega(P, Q)$ depend on the sign of the following six polynomials is deduced.

$$
\begin{aligned}
\Delta_{1}(e ; p, q)= & 2 p-2 q+e^{4} \\
\Delta_{2}(e ; p, q)= & p^{3}-3 p^{2} q-e^{8} q+3 p q^{2}+e^{8} p-2 e^{4} q^{2}-14 e^{4} q p-q^{3}-2 e^{4} p^{2} \\
\Delta_{3}(e ; p, q)= & p^{3}-3 p^{2} q-3 e^{4} p^{2}+3 p q^{2}-21 e^{4} q p+3 e^{8} p-3 e^{8} q-q^{3}-3 e^{4} q^{2}-e^{12} \\
\Delta_{4}(e ; p, q)= & 2-e^{2} \\
\Delta_{5}(e ; p, q)= & e^{2} p^{4}-p^{4}+4 p^{3} q-3 e^{2} p^{3} q+3 e^{2} p^{2} q^{2}-6 p^{2} q^{2}+4 p q^{3}-e^{2} p q^{3}-q^{4} \\
& +2 e^{4} p^{3}+p^{3}-e^{2} p^{3}-2 e^{6} p^{3}+4 e^{2} p^{2} q+14 e^{4} p^{2} q-3 p^{2} q-20 e^{6} p^{2} q \\
& -13 e^{4} p q^{2}-5 e^{2} p q^{2}-5 e^{6} p q^{2}+3 p q^{2}-q^{3}-3 e^{4} q^{3}+2 e^{2} q^{3}+e^{10} p^{2} \\
& -2 e^{4} p^{2}+2 e^{6} p^{2}-e^{8} p^{2}+e^{8} p q-14 e^{4} p q-4 e^{10} p q+21 e^{6} p q+4 e^{6} q^{2} \\
& -3 e^{8} q^{2}-2 e^{4} q^{2}+e^{8} p-e^{10} p-e^{12} q+2 e^{10} q-e^{8} q \\
\Delta_{6}(e ; p, q)= & \Delta_{3}(e ; p, q) \Delta_{0}(e ; p, q) .
\end{aligned}
$$

These polynomials are considered as bivariate polynomials in $p$ and $q$, whose coefficients depend on the eccentricity $e$. Geometrically, the equations $\Delta_{i}(e ; p, q)=0(i=1,2,3,5)$ represent planar implicit curves whose points have coordinates which depend on $e$. 
Observe that:

- the sign of $\Delta_{4}(e ; p, q)$ is $-($ if $e>\sqrt{2})$ or 0 (if $\left.e=\sqrt{2}\right)$

- As $\Delta_{0}(e ; p, q)>0, \operatorname{sign}\left(\Delta_{3}(e ; p, q)\right)=\operatorname{sign}\left(\Delta_{6}(e ; p, q)\right)$.

Thus, $\Omega(P, 1)$ and $\Omega(P, Q)$ really depend only on the signs of $\Delta_{i}(e ; p, q),(i=1,2,3,4,5)$. Let $R$ be the planar region defined by the conditions $p>0, q>0$ and $\Delta_{0}(e ; p, q)>0$ (displayed in Figure 4). In the following, $R$ is stratified according to the signs of the polynomials $\Delta_{i}(e ; p, q),(i=1,2,3,4,5)$.

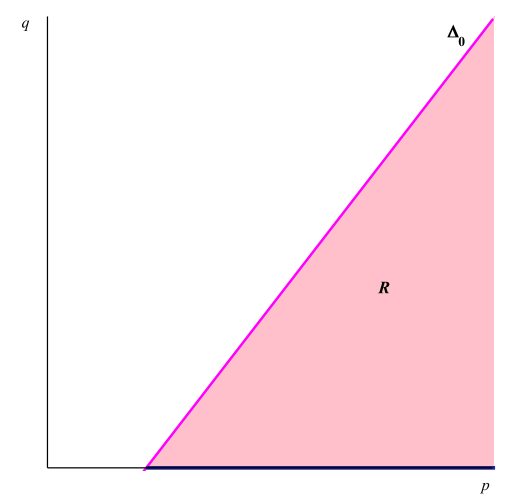

Figure 4: The region $R$ to be stratified and its borders: $\Delta_{0}=0$ (magenta) and $p$-axis, with $p \geq 1$ (navy)

The stratification is performed by determining (by means of geometrical and algebraical manipulations of the expressions $\Delta_{i}(e ; p, q)$ and its coefficients) the intersection points between the curve $\Delta_{i}(e ; p, q)=0$ and the borders of the region $R$, and between pairs of curves $\Delta_{i}(e ; p, q)=0, \Delta_{j}(e ; p, q)=0(j=1,2,3,5)$, for $i=1,2,3,5$. The results are presented in Table 1 , Figure 5 and Figure 6.

\begin{tabular}{|l|l|}
\hline Point & Belonging to \\
\hline $\mathbf{A}(1,0)$ & $p$-axis, $\Delta_{0}=0$ and $\Delta_{5}=0$ \\
$\mathbf{B}\left(e^{4}, 0\right)$ & $p$-axis, $\Delta_{2}=0, \Delta_{3}=0$ and $\Delta_{5}=0$ \\
$\mathbf{C}$ & $\Delta_{0}=0$ and $\Delta_{2}=0$ \\
$\mathbf{D}\left(\frac{3 e^{4}-4 e^{2}+2}{2 e^{2}\left(e^{2}-2\right)}, \frac{e^{8}-2 e^{6}+3 e^{4}-4 e^{2}+2}{2 e^{2}\left(e^{2}-2\right)}\right)$ & $\Delta_{0}=0$ and $\Delta_{1}=0$, if $e>\sqrt{2}$ \\
\hline
\end{tabular}

Table 1: Intersection points of the curves $\Delta_{i}=0,(i=1,2,3,5)$ in the region $R$.

Except for $\mathbf{D}$ (which exists only if $e>\sqrt{2}$ ), the existence of the obtained intersection points does not depend on the value of $e \geq \sqrt{2}$. Although the coordinates of the point $\mathbf{C}$ can't be explicitly expressed in terms of $e$, it is easy to prove (using, for instance, SturmHabicht coefficients and their properties) that the curves $\Delta_{0}(e ; p, q)=0$ and $\Delta_{2}(e ; p, q)=0$ has a unique intersection point in the positive quadrant.

In the following, the behavoiur of the curves $\Delta_{i}(e ; p, q)=0(i=1,2,3,5)$ in the region $R$ is explained. It is independent from the value of $e \geq \sqrt{2}$, except for $\Delta_{1}(e ; p, q)=0$ (whose behaviour has to be analyzed for $e>\sqrt{2}$ and for $e=\sqrt{2}$ ). In Figures 5 and 6 :

- The straight line $\Delta_{1}(e ; p, q)=0$ appears in red. If $e>\sqrt{2}$, it enters the region $R$ through the point $\mathbf{D}$. Otherwise (if $e=\sqrt{2}$ ), it doesn't enter the region $R$, as it is 
parallel with $\Delta_{0}(e ; p, q)=0$; in this case, it is displayed with a discontinue line, in order to highlight that it is not situated in the region of interest for us.

- The curve $\Delta_{2}(e ; p, q)=0$ appears in blue. It enters the region $R$ through the point $\mathbf{A}$, reaches again the $p$-axis at the point $\mathbf{B}$ (this curve segment is henceforth called the "left branch of $\Delta_{2}$ ") and afterwards remains in $R$ (this curve segment is henceforth called the "right branch of $\Delta_{2}$ ").

- The curve $\Delta_{3}(e ; p, q)=0$ appears in green. It enters the region $R$ through the point B.

- The curve $\Delta_{5}(e ; p, q)=0$ appears in cyan. It enters the region $R$ through the point $\mathbf{A}$, reaches again the $p$-axis at the point $\mathbf{B}$ (this curve segment is henceforth called the "left branch of $\Delta_{5}$ ") and afterwards remains in $R$ (this curve segment is henceforth called the "left branch of $\Delta_{5}$ ").

- The straight line $\Delta_{0}(e ; p, q)=0$ appears in magenta.

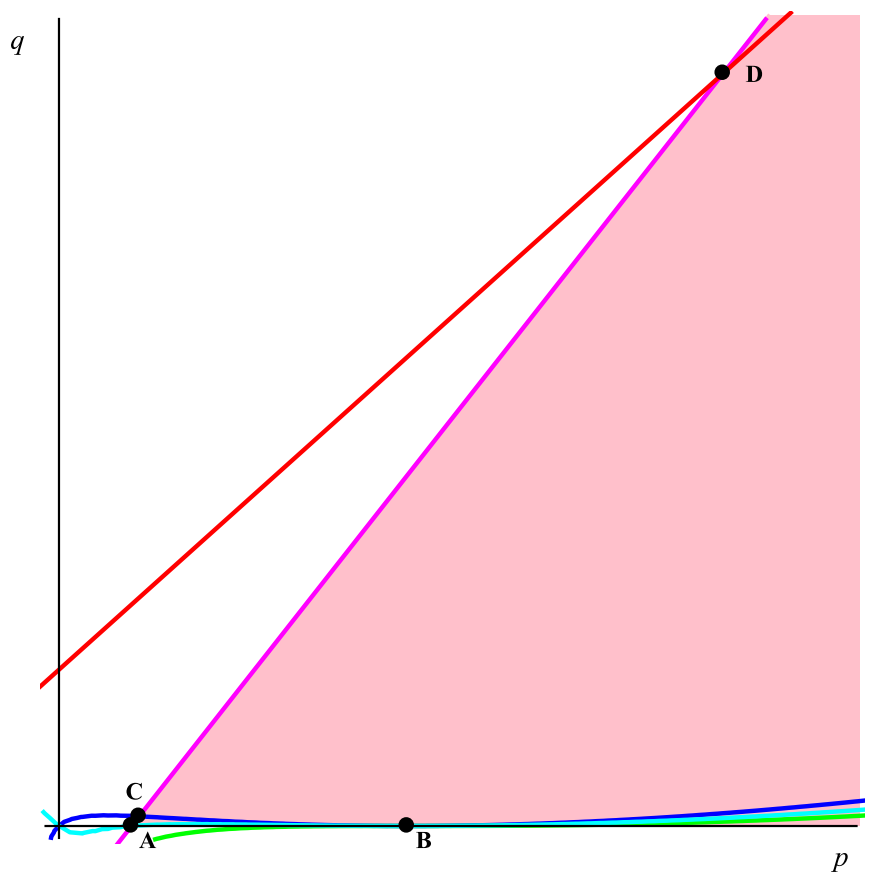

Figure 5: $\Delta_{1}=0$ (red), $\Delta_{2}=0$ (blue), $\Delta_{3}=0$ (green), $\Delta_{5}=0$ (cyan) and $\Delta_{0}=0$ (magenta), for $e>\sqrt{2}$.

By means of a point testing in each set (region or curve) generated by the aforementioned stratification, the signs of the polynomials stha ${ }_{j}(P)$ and stha ${ }_{j}(P, Q)$ (see Tables 5 and 4) are obtained as follows:

$$
\begin{gathered}
\operatorname{sign}\left(\left\{\mathbf{s t h a}_{j}(P)\right\}_{j \in\{4,3,2,1,0\}}\right)= \\
=\left\{+,+, \operatorname{sign}\left(\Delta_{1}(e ; p, q)\right), \operatorname{sign}\left(\Delta_{2}(e ; p, q)\right), \operatorname{sign}\left(\Delta_{3}(e ; p, q)\right)\right\},
\end{gathered}
$$

and

$$
\begin{gathered}
\operatorname{sign}\left(\left\{\operatorname{stha}_{j}(P, Q)\right\}_{j \in\{4,3,2,1,0\}}\right)= \\
\begin{cases}\left\{-,-,+, \operatorname{sign}\left(\Delta_{5}(e ; p, q)\right), \operatorname{sign}\left(\Delta_{3}(e ; p, q)\right)\right\} & \text { if } e>\sqrt{2} \\
\left\{-, 0,+, \operatorname{sign}\left(\Delta_{5}(e ; p, q)\right), \operatorname{sign}\left(\Delta_{3}(e ; p, q)\right)\right\} & \text { if } e=\sqrt{2} .\end{cases}
\end{gathered}
$$




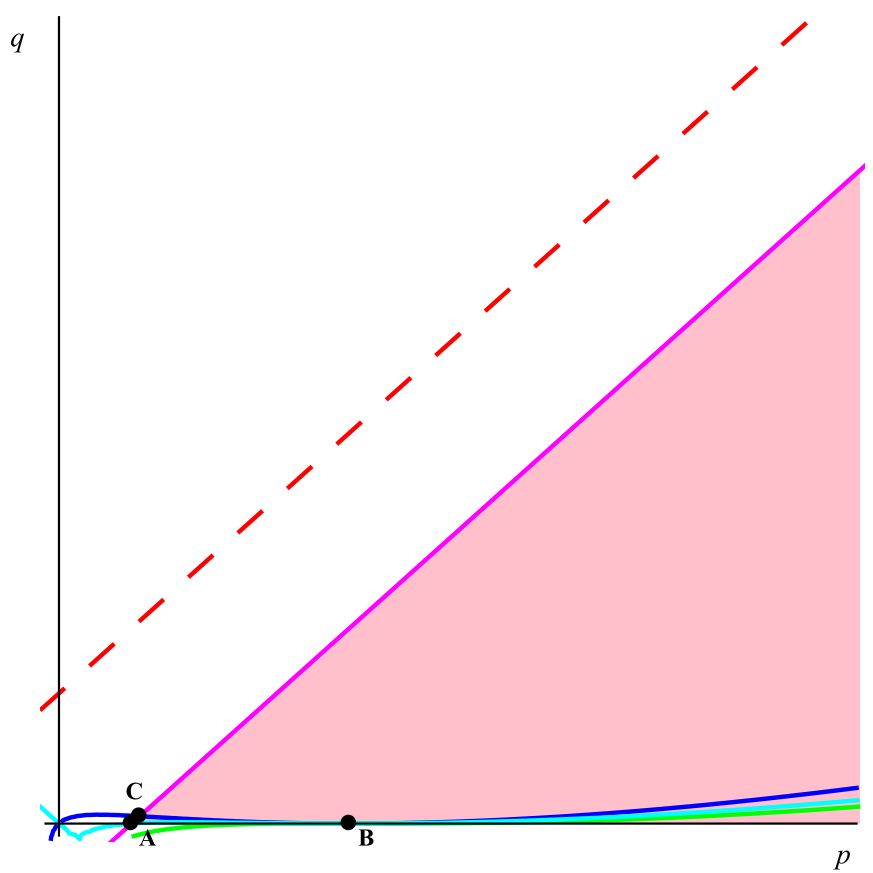

Figure 6: $\Delta_{1}=0$ (red), $\Delta_{2}=0$ (blue), $\Delta_{3}=0$ (green), $\Delta_{5}=0$ (cyan) and $\Delta_{0}=0$ (magenta), for $e=\sqrt{2}$.

As explained in Section 2, both the number of real roots of the polynomial $P(p, q, e ; k)$ which make $h$ positive and the number of real roots of $P(p, q, e ; k)$ which make $h$ negative are constant when $(p, q)$ belongs to any of the sets (regions or curves) generated by the stratification of the region $R$, provided by the curves $\Delta_{i}(e ; p, q)=0$. These curves divide the region $R$ into $n$ regions: $R_{1}, R_{2}, \ldots, R_{n}$, where $n=7$ if $e>\sqrt{2}$ and $n=6$ if $e=\sqrt{2}$ (see Figures 5, 6, 7 and Tables 3, 2).

The formulae (6) provide (as shown in Tables 5 and 4): $r$, the number of real roots of $P$ which make $h$ positive, and $s$, the number of real roots of $P$ which make $h$ negative (remember that these roots are counted without multiplicities).

The obtained results prove that, when the parameters $p$ and $q$ belong to the region $R$, the polynomial $P(e, p, q ; k)$ has a unique positive real root which makes $h$ positive:

- If $\Delta_{3}(e ; p, q)<0$ (in the region $R$ ), then $P(e, p, q ; k)$ has one positive real root which makes $h$ positive, $\alpha_{2}$.

- If $\Delta_{3}(e ; p, q)=0$ (in the region $R$ ), then $P(e, p, q ; k)$ has two real roots (counted without multiplicities) which make $h$ positive, the positive one $\alpha_{2}$ and the negative ones $\alpha_{3}=\alpha_{4}$.

- If $\Delta_{3}(e ; p, q)>0$ (in the region $R$ ), then $P(e, p, q ; k)$ has three real roots which make $h$ positive, the positive one $\alpha_{2}$, and the negative ones $\alpha_{3}$ and $\alpha_{4}$.

\section{$6 \quad$ Examples}

In this section, three examples are presented, corresponding to the three cases $\left(\Delta_{3}(e ; p, q)<\right.$ $0, \Delta_{3}(e ; p, q)=0$ and $\left.\Delta_{3}(e ; p, q)>0\right)$ studied in the Section 4.1. 

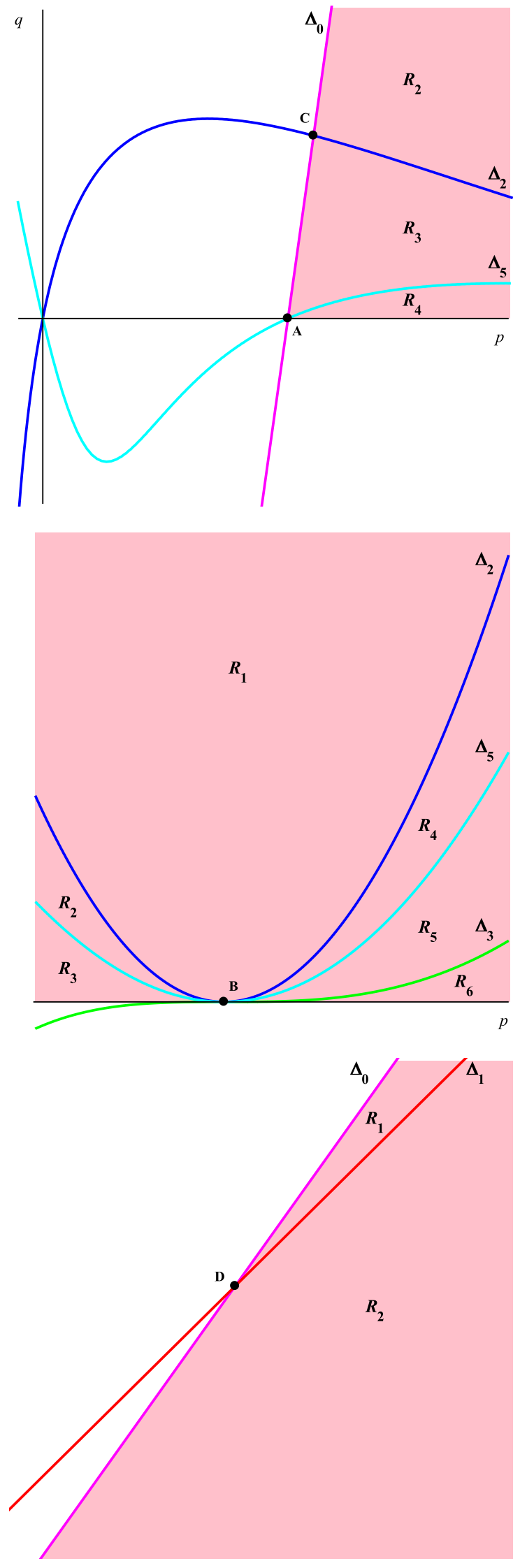

Figure 7: The curves $\Delta_{j}=0$ displayed around the points $\mathbf{A}$ and $\mathbf{C}$ (up), $\mathbf{B}$ (middle) and (for $e>\sqrt{2}) \mathbf{D}($ down). 


\begin{tabular}{|l|l|}
\hline Region $R_{i}$ & Its borders \\
\hline$i=1$ & $\begin{array}{l}\text { upper branch of } \Delta_{1} \\
\text { lower branch of } \Delta_{0}=0\end{array}$ \\
\hline$i=2$ & $\begin{array}{l}\text { lower branch of } \Delta_{1} \\
\text { upper branch of } \Delta_{2}=0 \\
\Delta_{0}=0\end{array}$ \\
\hline$i=3$ & $\begin{array}{l}p \in\left[1, e^{4}\right] \\
\text { upper branch of } \Delta_{5} \\
\text { lower branch of } \Delta_{2} \\
\Delta_{0}=0\end{array}$ \\
\hline$i=4$ & $\begin{array}{l}p \text {-axis with } p \in\left[1, e^{4}\right] \\
\text { lower branch of } \Delta_{5}\end{array}$ \\
\hline$i=5$ & $\begin{array}{l}\text { lower right branch of } \Delta_{2} \\
\text { upper right branch of } \Delta_{5}\end{array}$ \\
\hline$i=6$ & $\begin{array}{l}\text { lower right branch of } \Delta_{5} \\
\text { upper right branch of } \Delta_{3}\end{array}$ \\
\hline$i=7$ & $\begin{array}{l}p \text {-axis with } p \geq e^{4} \\
\text { lower branch of } \Delta_{3}\end{array}$ \\
\hline
\end{tabular}

Table 2: Description of the regions $R_{1}, R_{2}, \ldots, R_{7}$ for $e>\sqrt{2}$.

\begin{tabular}{|l|l|}
\hline Region $R_{i}$ & Its borders \\
\hline$i=1$ & $\begin{array}{l}p \geq 1 / 2+\sqrt{6} / 4 \\
\text { upper branch of } \Delta_{2} \\
\Delta_{0}=0\end{array}$ \\
\hline$i=2$ & $\begin{array}{l}p \in\left[1, e^{4}\right] \\
\text { upper branch of } \Delta_{5} \\
\text { lower branch of } \Delta_{2} \\
\Delta_{0}=0\end{array}$ \\
\hline$i=3$ & $\begin{array}{l}p \text {-axis with } p \in\left[1, e^{4}\right] \\
\text { lower branch of } \Delta_{5},\end{array}$ \\
\hline$i=4$ & $\begin{array}{l}\text { lower right branch of } \Delta_{2} \\
\text { upper right branch of } \Delta_{5}\end{array}$ \\
\hline$i=5$ & $\begin{array}{l}\text { lower right branch of } \Delta_{5} \\
\text { upper right branch of } \Delta_{3}\end{array}$ \\
\hline$i=6$ & $\begin{array}{l}p \text {-axis with } p \geq e^{4} \\
\text { lower branch of } \Delta_{3}\end{array}$ \\
\hline
\end{tabular}

Table 3: Description of the regions $R_{1}, R_{2}, \ldots, R_{6}$ for $e=\sqrt{2}$. 


\begin{tabular}{|l|l|l|l|l|}
\hline Set & $\operatorname{sign}\left(\mathbf{s t h a}_{j}(P)\right)$ & $\operatorname{sign}\left(\mathbf{s t h a}_{j}(P, Q)\right)$ & $r$ & $s$ \\
\hline Region $R_{1}$ & $\{+,+,-,-,-\}$ & $\{-,-,+,-,-\}$ & 1 & 1 \\
Region $R_{2}$ & $\{+,+,+,-,-\}$ & $\{-,-,+,-,-\}$ & 1 & 1 \\
Regions $R_{3}$ and $R_{5}$ & $\{+,+,+,+,-\}$ & $\{-,-+,-,-\}$ & 1 & 1 \\
Region $R_{4}$ and $R_{6}$ & $\{+,+,+,+,-\}$ & $\{-,-,+,+,-\}$ & 1 & 1 \\
Region $R_{7}$ & $\{+,+,+,+,+\}$ & $\{-,-,+,+,+\}$ & 3 & 1 \\
Curve $\Delta_{1}$ with $\Delta_{0}>0$ & $\{+,+, 0,-,-\}$ & $\{-,-,+,-,-\}$ & 1 & 1 \\
Curve $\Delta_{2}$ with $\Delta_{0}>0$ & $\{+,+, 0,-,-\}$ & $\{-,-,+,-,-\}$ & 1 & 1 \\
Curve $\Delta_{5}$ with $\Delta_{0}>0$ & $\{+,+,+,+,-\}$ & $\{-,-,+, 0,-\}$ & 1 & 1 \\
Curve $\Delta_{3}$ with $\Delta_{0}>0$ & $\{+,+,+,+, 0\}$ & $\{-, 0,+,+, 0\}$ & 2 & 1 \\
\hline
\end{tabular}

Table 4: Number of real roots of $P$ with $Q>0(r)$ and $Q<0(s)$, for $e>\sqrt{2}$.

\begin{tabular}{|l|l|l|l|l|}
\hline Set & $\operatorname{sign}\left(\mathbf{s t h a}_{j}(P)\right)$ & $\operatorname{sign}\left(\mathbf{s t h a}_{j}(P, Q)\right)$ & $r$ & $s$ \\
\hline Region $R_{1}$ & $\{+,+,+,-\}$, & $\{-, 0,+,-,-\}$ & 1 & 1 \\
Regions $R_{2}$ and $R_{4}$ & $\{+,+,+,+,-\}$ & $\{-, 0+,-,-\}$ & 1 & 1 \\
Region $R_{3}$ and $R_{5}$ & $\{+,+,+,+,-\}$ & $\{-, 0,+,+,-\}$ & 1 & 1 \\
Region $R_{6}$ & $\{+,+,+,+,+\}$ & $\{-, 0,+,+,+\}$ & 3 & 1 \\
Curve $\Delta_{2}$ with $\Delta_{0}>0$ & $\{+,+,+, 0,-\}$ & $\{-, 0,+,-,-\}$ & 1 & 1 \\
Curve $\Delta_{5}$ with $\Delta_{0}>0$ & $\{+,+,+,+,-\}$ & $\{-, 0,+, 0,-\}$ & 1 & 1 \\
Curve $\Delta_{3}$ with $\Delta_{0}>0$ and $p>e^{4}$ & $\{+,+,+,+, 0\}$ & $\{-, 0,+,+, 0\}$ & 2 & 1 \\
\hline
\end{tabular}

Table 5: Number of real roots of $P$ with $Q>0(r)$ and $Q<0(s)$, for $e=\sqrt{2}$. 
Remark 6. Obviously, the values appearing in these examples may be expressed exactly, using rational numbers and radicals, but huge written expressions (difficult to simplify) would be generated. For this reason, most of the values are displayed as floating point real numbers with four decimals.

Example with $\boldsymbol{\Delta}_{\mathbf{3}}(\mathbf{e} ; \mathbf{p}, \mathbf{q})<\mathbf{0}$. Consider the biaxial hyperboloid of one sheet defined by the semi axis $a=20, b=35$ and the point $(X, Y, Z)=(100,25,170)$, located out of the hyperboloid.

In this case, the eccentricity of the hyperboloid defined in the $X-Y$ plane is equal to $e=\frac{\sqrt{65}}{4}$ and the values of $p$ and $q$ are (see (11)):

$$
p=\frac{425}{16}, \quad q=\frac{14161}{64} .
$$

Observe that the point $(p, q)$ is placed in the region $R_{1}\left(\Delta_{1}(e ; p, q)<0, \Delta_{2}(e ; p, q)<0\right.$, $\left.\Delta_{3}(e ; p, q)<0, \Delta_{4}(e ; p, q)<0, \Delta_{5}(e ; p, q)<0\right)$ and hence the polynomial $P$ has two positive real roots and two complex roots,

$$
\begin{aligned}
& \alpha_{1}=k_{1}=5.9867, \\
& \alpha_{2}=k_{2}=3.0328, \\
& \alpha_{3}=k_{3}=-0.4473+I 14.1746, \\
& \alpha_{4}=k_{4}=-0.4473-I 14.1746 .
\end{aligned}
$$

Using the smallest positive root, $\alpha_{2}=k_{2}$, the hyperboloidal coordinates of the point $(X, Y, Z)$ are (see $(15))$

$$
\begin{aligned}
h & =3.4007, \\
\lambda & =0.2449, \\
\phi & =0.5104 .
\end{aligned}
$$

Example with $\boldsymbol{\Delta}_{\mathbf{3}}(\mathbf{e} ; \mathbf{p}, \mathbf{q})>\mathbf{0}$. Consider the biaxial hyperboloid of one sheet defined by the semi axis $a=100, b=10$ and the point $(X, Y, Z)=(150,120,12)$, located out of the hyperboloid.

In this case, the eccentricity of the hyperboloid defined in the $X-Y$ plane is $e=\frac{\sqrt{101}}{10}$. The values of $p$ and $q$ are (see (11)):

$$
p=\frac{369}{100}, \quad q=\frac{9}{62500} .
$$

The polynomial $P$ has two positive and two negative roots,

$$
\begin{aligned}
& \alpha_{1}=k_{1}=2.9309, \\
& \alpha_{2}=k_{2}=0.0073, \\
& \alpha_{3}=k_{3}=-0.0074, \\
& \alpha_{4}=k_{4}=-0.9107 .
\end{aligned}
$$

Using the smallest positive root, $\alpha_{2}=k_{2}$, the hyperboloidal coordinates of the point $(X, Y, Z)$ are (see (15)):

$$
\begin{aligned}
h & =4.3713, \\
\lambda & =0.6747, \\
\phi & =1.4540 .
\end{aligned}
$$


Example with $\boldsymbol{\Delta}_{\mathbf{3}}(\mathbf{e} ; \mathbf{p}, \mathbf{q})=\mathbf{0}$. Consider the biaxial hyperboloid of one sheet defined by the semi axis $a=100, b=100$ and the point $(X, Y, Z)=(200,364.9894,100)$, located out of the hyperboloid.

In this case, the eccentricity of the hyperboloid defined in the $X-Y$ plane is $e=\sqrt{2}$. The values of $p$ and $q$ are (see (11)):

$$
p=17.3217, \quad q=1 .
$$

Observe that the point $(p, q)$ is placed in the region $\Delta_{3}(e ; p, q)=0$, with $\Delta_{0}(e ; p, q)>0$ $\left(\Delta_{1}(e ; p, q)>0, \Delta_{2}(e ; p, q)>0, \Delta_{3}(e ; p, q)=0, \Delta_{4}(e, p, q)=0, \Delta_{5}(e ; p, q)>0\right)$ and hence the polynomial $P$ has two positive and two negative roots,

$$
\begin{aligned}
& \alpha_{1}=k_{1}=6.1072, \\
& \alpha_{2}=k_{2}=0.4125, \\
& \alpha_{3}=k_{3}=-1.2598, \\
& \alpha_{4}=k_{4}=\alpha_{3} .
\end{aligned}
$$

Using the smallest positive root, $\alpha_{2}=k_{2}$, the hyperboloidal coordinates of the point $(X, Y, Z)$ are $($ see $(15))$ :

$$
\begin{aligned}
h & =209.7298, \\
\lambda & =1.0695, \\
\phi & =0.7461 .
\end{aligned}
$$

\section{Conclusions}

In our paper, a direct transformation from cartesian coordinates into hyperboloidal coordinates (considered for biaxial hyperboloids) has been presented. Its practical significancy resides in applications in the Geodesy field, being of interest in hyperboloidal building and cooling tower construction.

At our knowledge, the issue of transforming the Cartesian coordinates into hyperboloidal coordinates has been treated so far only in [2], where the presented solutions are based on numerical iteration processes.

Our approach, based on symbolic techniques, improves the solution presented in [2], as it is reduced to a few mere evaluations of symbolic expressions. Given the 3D cartesian coordinates of a point located out from a hyperboloidal surface, the coordinate transformation process consists only in evaluating:

- the positive root $\alpha_{2}($ see $(25))$ (or $k_{2}$ (see (31)),

- the distance $D$ (see (13)) and

- the hyperboloidal coordinates (see (15)).

Consequently, it is very accurate and (without having computing the complexities of the

algorithms presented in [2] and for sake of the utmost correctness) highly presumable to be less time consuming: our solution has been generically (i.e. symbolically) computed once, as presented in this paper, and remains at any user's disposal for further application (i.e. evaluations); moreover, the precision of the aforementioned evaluations may be chosen by the user.

The transformation problem has been reduced to the problem of finding the positive roots of a fourth degree polynomial and the analysis of the polynomial's roots has been 
performed by an algebraically complete stratification of a planar region situated in the positive quadrant. In order to compute the polynomial's roots, two approaches have been presented: one based on the Merriman method and the other one obtained using the Computer Algebra System Maple, which applies the Ferrari method. In this sense, a conjecture concerning the algebraical equalness of the roots has been proposed to the readers.

One of the main topics of our further work consists in studying the case of the hyperboloidal coordinates considered for triaxial hyperboloids and providing a similar (symbolic) approach for the tranformation of the cartesian coordinates.

\section{Acknowledgments}

Prof. Laureano Gonzalez-Vega is kindly thanked for suggesting us the problem and discussing it with us, by devoting time and support, and sharing with us his expertise and research insight.

The authors are partially supported by the Spanish "Ministerio de Economía y Competitividad" and by the European Regional Development Fund (ERDF), under the Project MTM2011-25816-C02-02. The second author is partially supported by the European Project SAGA (FP7-PEOPLE-2007-1-1-ITN).

The reviewers are warmly thanked for their suggestions and comments which helped us to improve this paper.

\section{References}

[1] S. Basu, R. Pollack, MF. Roy. Algorithms in Real Algebraic Geometry. Algorithms and Computations in Mathematics, 10, second ed., Springer, Berlin, 2006.

[2] J. Feltens. Hyperboloidal coordinates: transformations and applications in special constructions. Journal of Geodesy, 85 (4) (2011), 239-254.

[3] L. Gonzalez-Vega, H. Lombardi, T. Recio, MF. Roy. Sturm-Habicht Sequences, Determinants and Real Roots of Univariate Polynomials, in: B.F. Caviness, J.R. Johnson (Eds.), Quantifier Elimination and Cylindrical Algebraic Decomposition. Texts and Monographs in Symbolic Computation, Springer, Berlin, 1998, pp. 300316.

[4] L. Gonzalez-Vega, I. Polo-Blanco. A symbolic analysis of Vermeille and Borkowski polynomials for transforming 3D Cartesian to geodetic coordinates. Journal of Geodesy, 83 (11) (2009), 1071-1081.

[5] M. Merriman. The deduction of final formulas for the algebraic solution of the quartic equation. American Journal of Mathematics, 14 (3) (1892), 237-245.

[6] H. Vermeille. Direct transformation from geocentric coordinates to geodetic coordinates. Journal of Geodesy, 76 (8) (2002), 451-454. 